\title{
Black Holes and Wormholes in Extended Gravity
}

\author{
Stanislav Alexeyev ${ }^{1,2, *}$ and Maxim Sendyuk ${ }^{3}$ \\ 1 Sternberg Astronomical Institute, Lomonosov Moscow State University, Universitetskii Prospekt, 13, \\ 119234 Moscow, Russia \\ 2 Department of Quantum Theory and High Energy Physics, Physics Faculty, Lomonosov Moscow State \\ University, Leninskie gory, 1/2, 119234 Moscow, Russia \\ 3 Department of Astrophysics and Stellar Astronomy, Physics Faculty, Lomonosov Moscow State University, \\ Leninskie gory, 1/2, 119234 Moscow, Russia; sendiuk.ms16@physics.msu.ru \\ * Correspondence: alexeyev@physics.msu.ru
}

Received: 14 December 2019; Accepted: 23 January 2020; Published: 27 January 2020

\begin{abstract}
We discuss black hole type solutions and wormhole type ones in the effective gravity models. Such models appear during the attempts to construct the quantum theory of gravity. The mentioned solutions, being, mostly, the perturbative generalisations of well-known ones in general relativity, carry out additional set of parameters and, therefore could help, for example, in the studying of the last stages of Hawking evaporation, in extracting the possibilities for the experimental or observational search and in helping to constrain by astrophysical data.
\end{abstract}

Keywords: general relativity; Gauss-Bonnet gravity; string effective action; brane world; Brans-Dicke model; black hole; wormhole

PACS: 04.50.+h; 04.50.Gh; 04.80.Cc

\section{Introduction}

The idea to construct the quantum theory of gravity leads to the appearance of a set of new models, for example, effective ones with scalar field(s), models with higher order curvature corrections, non-compact extra-dimensions and so on. In such a way, the Brans-Dicke theory often appears to be the first step in extending the general relativity (GR) [1] and, even at this level, new intriguing properties occur. Furthermore, the construction of the effective quantum gravity action leads to an extension of Einstein-Hilbert one by, for example, higher order curvature corrections [2]. As one usually begins from the first expansion terms, the investigation of the second order curvature corrections becomes important. According to Ref. [3], their most physically interesting form includes the Gauss-Bonnet invariant [4]. In the modern models, it is coupled with the scalar field to make the contribution of Gauss-Bonnet term be dynamical. This model appeared to be very fruitful because the order of field equations remains the second one; therefore, the transition to GR is provided.

Since 1938, the idea to extend GR by higher-order curvature terms has been developing $[4,5]$. In Ref. [3], the form of the action extended by the second order curvature corrections was suggested and proved. Taking into account the string theory effective action expansions, the most generic case of the gravitational Lagrangian appears to be as follows [6]:

$$
L=\sqrt{-g}\left(R-2 \Lambda+\alpha_{2} L_{2}+\alpha_{3} L_{3}+\ldots\right),
$$


where $g$ is the metrics determinant, $R$ is the Ricci scalar, $\Lambda$ is the cosmological constant, $L_{i}$ are $i$-th higher order curvature corrections, and $\alpha_{i}$ are the corresponding coupling constants. The second-order curvature correction is based on the Gauss-Bonnet invariant:

$$
S_{G B}=R_{\mu \nu \lambda \sigma} R^{\mu \nu \lambda \sigma}-4 R_{\mu v} R^{\mu v}+R^{2} .
$$

Firstly, the Gauss-Bonnet term appeared in the attempts to obtain quantum gravity as as counter term during the theory renormalization procedure [2]. After the string theory was developed, such an action began to serve as string theory effective one for gravity description [6]. The consideration of a theory with higher-order curvature corrections with the Lagrangian (1) as an effective low-energy limit of string theory leads to the action in the form:

$$
S=\frac{1}{2} \int d^{n} x \sqrt{-g}\left(-R+2 \partial_{\mu} \phi \partial^{\mu} \phi-e^{-2 \phi} F_{\mu v} F^{\mu v}+\lambda e^{-2 \phi} S_{G B}+\ldots\right),
$$

where $\phi$ is the dilatonic field making the contribution of Gauss-Bonnet term be dynamical (and originating from string/M theory); $F_{\mu \nu}$ is the Maxwell's tensor.

The discussed types of effective actions provide the local ones in addition to global cosmological solutions [7]. The most remarkable of them are of black hole type and wormhole one. These solutions expand the well-known Schwarzschild space-time and demonstrate something new that we recover in this paper. Here, It is important to note that in this model the new black hole solutions extend the well-known GR Schwarzschild and Kerr metrics, but, in contradiction, with no-hair theorem introducing new types of black hole characteristics. In these string gravity effective models, the consideration often is restricted by the second order curvature correction. In such model, various stationary spherically-symmetric solutions describing black hole-like objects were obtained [7-9].

The going on to $N>4$ space-time allows for neglecting the scalar field (as the Gauss-Bonnet term would no longer be total derivative) but preserves such interesting solution feathers. The particular $6 \mathrm{D}$ case is also considered. Furthermore, we try to extend the consideration to a more complicated case wormhole solution (but it occurred that it is possible to restrict the consideration by simpler models).

One of the important questions that we pay attention for the entire paper is the possibility to observe the solution in the nature. New results in high energy physics and astronomy provide additional possibilities to distinguish different types of theoretical solutions.

The paper is organised as follows. Section 2 is devoted to the non-rotating black hole solutions in Gauss-Bonnet extended gravity including the four-dimensional case (Section 2.1), multidimensional case (Section 2.3) and particular case: Dadhich-Molina solution. Section 3 generalises the discussion and includes the results of black hole solutions properties in effective equations of the Randall-Sundrum model. Section 4 switches to wormhole solutions in a more simple case: the Brans-Dicke model because even at this level some interesting particularities appear. Thus, in Section 4.1, we discuss non-rotating wormhole solutions and, in Section 4.1, we extend the discussion to the de Sitter universe. Section 5 contains our conclusions.

\section{Dilatonic (Einstein-Maxwell-)Gauss-Bonnet Gravity}

\subsection{D Black Hole Solutions in Gauss-Bonnet Gravity}

A four-dimensional (4D) black hole solution in Gauss-Bonnet gravity was presented in the form of power series [10,11] and in the numerical one [8]. However, this solution was found only outside the horizon. A common description of inner and outer structures was presented in Ref. [7]. Thus, the gravitational action was taken as:

$$
S=\frac{1}{2 \kappa} \int d^{4} x \sqrt{-g}\left(m_{p l}^{2}\left(-R+2 \partial_{\mu} \phi \partial^{\mu} \phi\right)+\lambda e^{-2 \phi} S_{G B}\right),
$$


where $m_{p l}$ is the Planck mass.

The metric could be chosen in the curvature coordinate form as

$$
d s^{2}=\Delta c^{2} d t^{2}-\frac{\sigma^{2}}{\Delta} d r^{2}-r^{2}\left(d \theta^{2}+\sin ^{2} \theta d \varphi^{2}\right),
$$

where $\Delta$ and $\sigma$ depend on radial coordinates $r$ only. The corresponding Einstein equations are:

$$
\begin{aligned}
& m_{p l}^{2} \sigma^{2}\left[-r \sigma^{\prime}+\sigma r^{2}\left(\phi^{\prime}\right)^{2}\right]+4 e^{-2 \phi} \lambda \sigma\left(\Delta-\sigma^{2}\right)\left[\phi^{\prime \prime}-2\left(\phi^{\prime}\right)^{2}\right]+4 e^{-2 \phi} \phi^{\prime} \lambda \sigma^{\prime}\left(\sigma^{2}-3 \Delta\right)=0, \\
& m_{p l}^{2} \sigma^{2}\left[\sigma^{2}+\Delta r^{2}\left(\phi^{\prime}\right)^{2}-\Delta^{\prime} r-\Delta\right]+4 e^{-2 \phi} \phi^{\prime} \lambda \Delta^{\prime}\left(\sigma^{2}-3 \Delta\right)=0, \\
& m_{p l}^{2} \sigma^{2}\left[\Delta^{\prime \prime} r \sigma-\Delta^{\prime} r \sigma^{\prime}+2 \Delta^{\prime} \sigma-2 \Delta \sigma^{\prime}-2 r \Delta \sigma\left(\phi^{\prime}\right)^{2}\right]+8 e^{-2 \phi} \lambda\left\{\sigma \Delta \Delta^{\prime}\left[\phi^{\prime \prime}-2\left(\phi^{\prime}\right)^{2}\right]\right. \\
+ & \left.\phi^{\prime}\left[\left(\Delta^{\prime}\right)^{2} \sigma+\Delta \Delta^{\prime \prime} \sigma-3 \Delta \Delta^{\prime} \sigma^{\prime}\right]\right\}=0, \\
& -2 m_{p l}^{2} \sigma^{2}\left[\Delta^{\prime} r^{2} \sigma \phi^{\prime}+2 r \Delta \phi^{\prime} \sigma-r^{2} \Delta \phi^{\prime} \sigma^{\prime}+r^{2} \Delta \phi^{\prime \prime} \sigma\right] \\
+ & 4 e^{-2 \phi} \lambda\left[\left(\Delta^{\prime}\right)^{2} \sigma+\Delta \Delta^{\prime \prime} \sigma-3 \Delta \Delta^{\prime} \sigma^{\prime}-\Delta^{\prime \prime} \sigma^{3}+\Delta^{\prime} \sigma^{\prime} \sigma^{2}\right]=0 .
\end{aligned}
$$

This system was solved numerically and the solutions are presented in Figure 1.
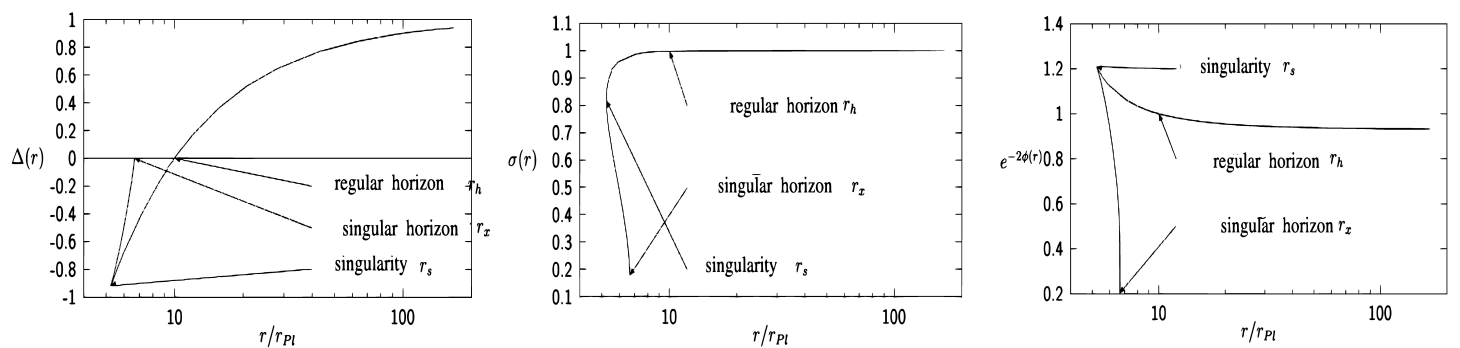

Figure 1. Dependence of the metric functions $\Delta, \sigma$ and the dilatonic exponent $e^{-2 \phi}$ against the radial coordinate $r$. Reproduced from [12].

Outside the horizon, the metric functions and the dilatonic one behave in the ordinary Schwarzschild-like way, whereas, inside the horizon, they exist only until the singularity at $r=r_{s}$. The solution includes the additional branch starting at $r_{s}$ (see Figure 1). This branch finishes at $r_{x}$, hence it is non-physical [12]. Since the Kretchman scalar diverges at this point:

$$
R_{\mu v \rho \sigma} R^{\mu \nu \rho \sigma} \propto\left(r-r_{x}\right)^{-5} \rightarrow \infty,
$$

$r_{x}$ represents an inner singular horizon. The distance between $r_{x}$ and $r_{h}$ decreases with $r_{h}$ decreasing. The boundary case $r_{s}=r_{x}=r_{h}$ represents a lower limit of the possible horizon radius value [13]:

$$
r_{h}^{\min }=\sqrt{4 \sqrt{6}} \frac{\sqrt{\lambda}}{m_{p l}} .
$$

The existence of this lower limit is specific for Gauss-Bonnet gravity (and some other extended model [9]) and does not appear in pure GR.

Here, it is necessary to point out that the interest to Gauss-Bonnet black holes regularly persists and increases. Thus, during the last few years, these results were re-obtained in the the framework of a more generic model including cosmological constant [14]. As in Ref. [8], the obtained limits are treated as constraints on dilatonic function. A wider set of dilatonic coupling functions (see, also $[15,16]$ ) is studied. It is demonstrated that the behaviour of dilatonic function at large distances appears to be growing because of cosmological constant influence. 
This black hole solution can be generalised by the Maxwell tensor [17]. Therefore, the action is rewritten as

$$
S=\frac{1}{2 \kappa} \int d^{4} x \sqrt{-g}\left(m_{p l}^{2}\left(-R+2 \partial_{\mu} \phi \partial^{\mu} \phi\right)-e^{-2 \phi} F_{\mu v} F^{\mu v}+\lambda e^{-2 \phi} S_{G B}\right) .
$$

A convenient choice of metric gauge now is:

$$
d s^{2}=\Delta c^{2} d t^{2}-\frac{1}{\Delta} d r^{2}-f^{2}\left(d \theta^{2}+\sin ^{2} \theta d \varphi^{2}\right),
$$

where $\Delta$ and $f$ are the functions against $r$ only. The presence of a magnetic charge was under investigation, thus the ansatz for the Maxwell tensor is:

$$
F=q \sin \theta d \theta \wedge d \varphi .
$$

The solution asymptotically corresponds to the Gibbons-Maeda-Garfinkle-Horowitz-Strominger (GM-GHS) one [18]. Depending upon the charge, the solution can differ from the previous one (plotted in Figure 1) significantly. As the charge exceeds its critical value $q_{c r}$, the inner singularity at $r_{s}$ disappears. This mechanism appears to be the same as in wormholes (the disappearing of the inner singularity transfers the black hole into the wormhole) or in non-singular cosmology when the initial singularity becomes a bounce. Figure 2 shows this behaviour with the numerical solution.
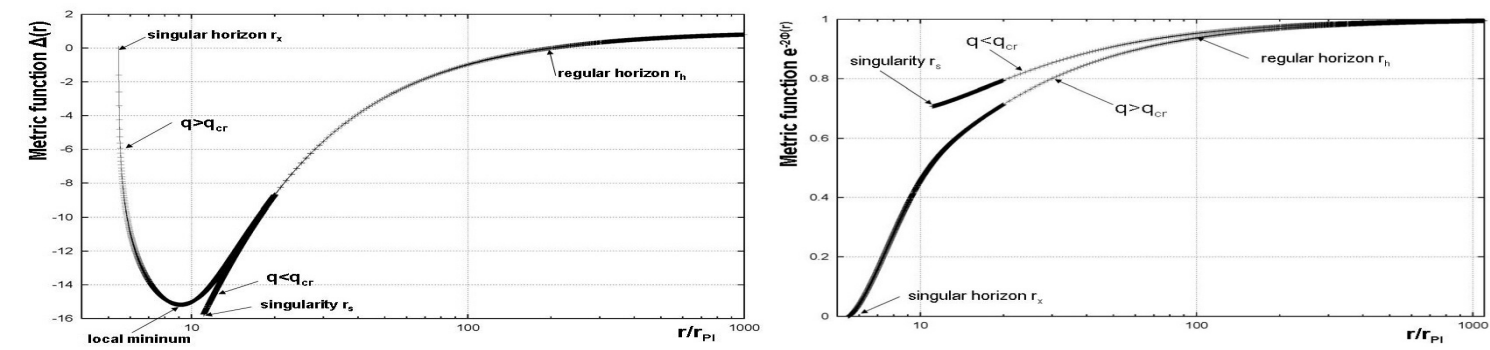

Figure 2. The dependence of the metric function $\Delta$ and the dilatonic one $e^{-2 \phi}$ upon the radial coordinate $r$ for $q<q_{c r}$ (left curve) and $q=24.81>q_{c r}$ (right one). Reproduced from [17].

The evaporation of the Gauss-Bonnet black holes was also presented in [12,19]. The existence of the lower limit on black hole radii (or mass) (8) leads to an interesting behaviour at final evaporation stages, when the second-order curvature terms contribute significantly. In contradiction with the usual picture, the evaporation rate changes to:

$$
-\frac{d M}{d t}=\int_{0}^{\left(M-M_{\min }\right) c^{2}} \frac{\Gamma_{s}(M, E)}{2 \pi \hbar} \frac{\Theta\left[\left(M-M_{\min }\right) c^{2}-E\right]}{e^{\operatorname{Im}(S)}-(-1)^{2 s}} \frac{E}{c^{2}} d E,
$$

where $M_{\min } \sim 10 m_{p l}$ is the minimal mass of a black hole, $\Gamma_{s}(M, E)$ is the probability of the absorption of particles with the spin $s, \Theta$ is the Heavyside step function, and $S$ is the action of the particle that is tunneling though the horizon potential barrier. In order to study the final stages, one has to study the first order expansion of $e^{\operatorname{Im}(S)}$. The evaporation rate-mass dependence is presented in Figure 3. The final stages differ from the Hawking ones. There is a maximum of the mass lost rate at the point close to the minimal mass $\left(\sim\left(10-10^{3}\right) m_{p l}\right)$ which considerably exceeds the evaporation rate for these masses in Hawking's law. Then, the process of the evaporation stops. 


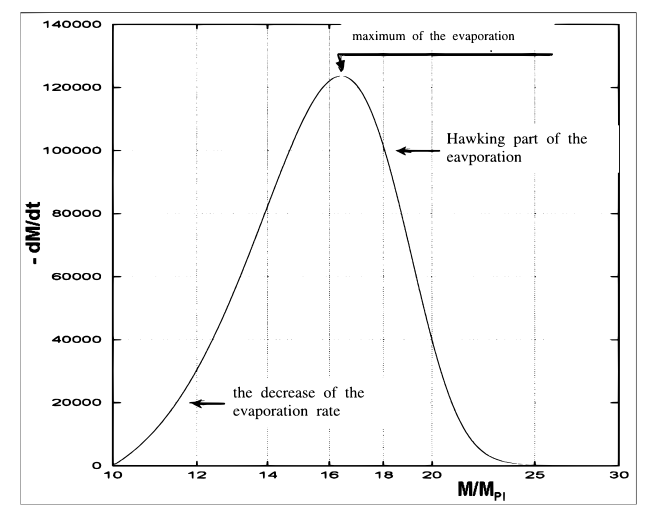

Figure 3. The mass lost rate of a Gauss-Bonnet black hole. The right part of the curve is the same as the ordinary Hawking evaporation law $-\frac{d M}{d t} \propto \frac{1}{M^{2}}$. The left part of the curve represents a decrease of the evaporation rate and stopping of the process at $M_{\text {min }}$ (It does not occur in GR). Reproduced from [12].

This model has two important consequences. Firstly, it predicts a strong flash near the maximum. These flashes can be the origin of high-energy cosmic rays, so a certain part of observed high-energy cosmic rays could originate from evaporating Gauss-Bonnet black holes at final stages. Secondly, there is a non-zero final mass, when the evaporation stops. As a consequence, an extremely weakly interacting (with a cross section $\sim 10^{-70} \mathrm{~m}^{2}$ ) massive object is formed. This object can pass through a neutron star without interaction [12]. Such objects could be an alternative to dark matter, explaining the irregular dynamics in galaxies.

During the last few years, the interest in Gauss-Bonnet black hole solutions resumed due to the ideas of scalarization [20]. In this context, these solutions were studied in more detailed form, including the presence of a cosmological constant [14], the presence of the massive scalar field [21,22], and different forms of coupling functions [21,23-25]. It was shown that the near-horizon geometry in these black hole solutions becomes more complicated with new constraints on scalar field asymptotic value $\phi_{\infty}$ arise; therefore, the extraction of a minimal black hole mass appears to not be so evident. The stability of the solutions was also studied [26], and, nowadays, it is proved much more carefully in a wide range [27-29].

\subsection{Multidimensional Non-Rotating Black Hole Solutions in Gauss-Bonnet Gravity}

Multidimensional Schwarzschild-like black holes embedded in the anti de-Sitter (AdS) Universe in the framework of Gauss-Bonnet gravity were studied in [30]. The action was identical to (17) except for an ordinary Einstein constant in the place of a six-dimensional one. In $D$ dimensions, the cosmological constant is written as follows:

$$
\Lambda=\frac{-(D-1)(D-2)}{2 l^{2}},
$$

where $l$ is the AdS radius.

The solution obtained in [30] is:

$$
d s^{2}=e^{2 v} c^{2} d t^{2}-e^{2 \mu} d r^{2}-r^{2} h_{i j} d x^{i} d x^{j},
$$

where

$$
e^{2 v}=e^{-2 \mu}=1+\frac{r^{2}}{2 \alpha(D-3)(D-4)}\left(1 \pm \sqrt{1+\frac{32 \pi^{\frac{3 D}{2}} G \alpha(D-3)(D-4) M \Gamma\left(\frac{D-1}{2}\right)}{c^{2}(D-2) r^{D-1}}}\right)
$$


$h_{i j} d x^{i} d x^{j}$ is a line element of a $(D-2)$-dimensional hyper space-time, $G$ is the $D$-dimensional gravitational constant, $M$ is a mass, and $\Gamma$ is the gamma-function,

$$
M=\frac{(D-2) \pi^{\frac{D-1}{2}} r_{+}^{D-3} c^{2}}{8 \pi G \Gamma\left(\frac{D-1}{2}\right)}\left(1+\frac{a(D-3)(D-4)}{r_{+}^{2}}\right),
$$

where $r_{+}$is the outer horizon radius.

The temperature of a multidimensional non-rotating black hole was calculated in [31]. The temperature ratio Gauss-Bonnet black hole to the Schwarzschild one is illustrated in Figure 4. Sometimes, the difference between the temperature of a GR black hole and a Gauss-Bonnet one can be considerably large, and even exceed 5\%, which makes these types of black holes experimentally distinguishable.
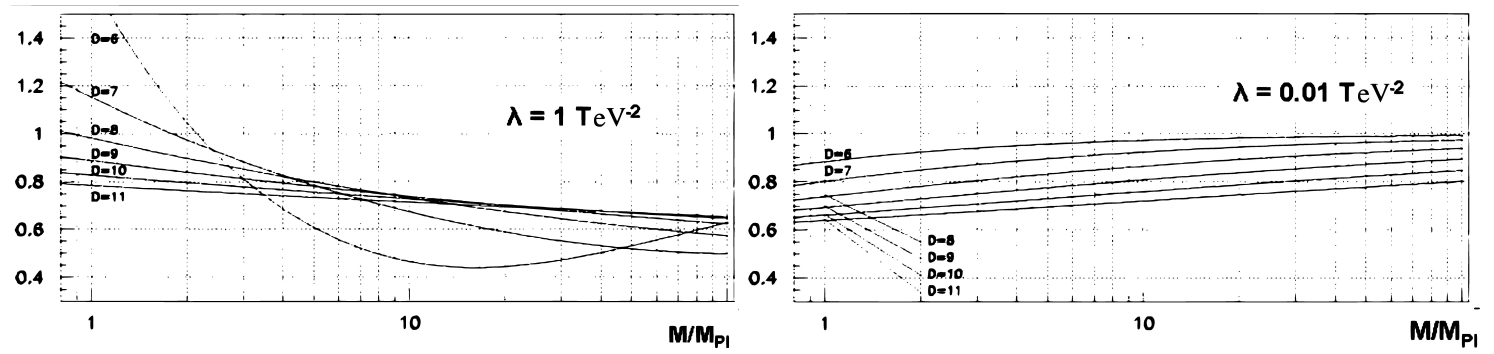

Figure 4. Ratio of the temperatures with and without the Gauss-Bonnet term as a function of mass. Reproduced from [12].

It is worth mentioning that this difference for a rotating Gauss-Bonnet black hole is below 5\% [32], and is thus non-observable, but, according to [33], an evaporating black hole loses its angular momentum very rapidly and soon can be considered as non-rotating.

\subsection{Dadhich-Molina 6D-Solution}

Developing string gravity effective actions with the higher order curvature corrections, Maeda and Dadhich presented a solution in a $N>4$ space-time being a product of the usual $4 \mathrm{D}$ one and a (n-4)-dimensional space with constant negative curvature [34-36]. Furthermore, Dadhich and Molina [37] considered the particular case: a minimal 6D of Maeda-Dadhich (DM) solution. The gravitational action with the Gauss-Bonnet curvature correction used is:

$$
S=\int d^{6} x \sqrt{-g}\left[\frac{1}{2 \kappa_{6}}\left(R-2 \Lambda+\alpha L_{G B}\right)\right]
$$

where $\kappa_{6}$ is $6 \mathrm{D}$ Einstein constant, $\alpha$ is the Gauss-Bonnet coupling one, and $L_{G B}$ is the Gauss-Bonnet term. Since the number of dimensions does not exceed six, there is no contribution from the cubic curvature term [3]. Hence, in the DM case, one has a right to consider the Gauss-Bonnet gravity as a precise model. In [37], the coupling constant $\alpha$ is considered to be positive or equal to zero. Such an action leads to the following field Equations:

$$
G^{\mu}{ }_{v}+\alpha H^{\mu}{ }_{v}+\Lambda \delta^{\mu}{ }_{v}=\kappa_{6} T^{\mu}{ }_{v},
$$

where $G_{\mu v}$ is the Einstein tensor, $T_{\mu \nu}$ is energy-momentum one and

$$
H_{\mu \nu}=2\left(R R_{\mu \nu}-2 R_{\mu \sigma} R^{\sigma}{ }_{v}-2 R^{\sigma \rho} R_{\mu \sigma \nu \rho}+R_{\mu}{ }^{\sigma \rho \beta} R_{\nu \sigma \rho \beta}\right)-\frac{1}{2} g_{\mu v} L_{G B} .
$$


In the vacuum case where $T_{\mu v}=0$ and assuming that the $6 \mathrm{D}$ space-time is homeomorphic to $M^{4} \times K^{2}$ where $M^{4}$ is a four-dimensional physical space-time and $K^{2}$ is a two-dimensional space of constant curvature, it is possible restrict the consideration with the single scalar equation instead of (18):

$$
\stackrel{(4)}{R}+\alpha L_{G B}^{(4)}+\frac{1}{2 \alpha}=0,
$$

where four-dimensional quantities are denoted with "(4)". Equation (20) has the following static spherically-symmetric solution:

$$
d s^{2}=f(r) c^{2} d t^{2}-\frac{1}{f(r)} d r^{2}-r^{2} d \Omega^{2}
$$

where

$$
f(r)=1+\frac{r^{2}}{4 \alpha}\left[1 \pm \sqrt{\frac{2}{3}+16\left(\frac{\alpha^{\frac{3}{2}} M}{r^{3}}-\frac{\alpha^{2} q}{r^{4}}\right)}\right]
$$

and $M$ and $q$ are arbitrary dimensionless constants.

This solution was studied in [38] with the asymptotically anti-De Sitter (AdS) metric. Thus, the asymptotic behaviour of $f(t)$ metric function at $r \rightarrow \infty$ is:

$$
f(r)=1 \pm \frac{2 \sqrt{\frac{3 \alpha}{2}} M}{r} \mp \frac{\sqrt{6} \alpha q}{r^{2}}+\frac{3 \pm \sqrt{6}}{12} \frac{r^{2}}{\alpha} .
$$

The left-hand side of the effective Einstein's equations is well-determined, whereas the exact form of the energy-momentum tensor, which is formed out of the induced matter, usually is not known precisely. Nonetheless, using the superpotential technique, which requires only geometric properties of the effective GR space-time, it becomes possible to calculate the total energy:

$$
E= \pm \sqrt{\frac{3 \alpha}{2}} M
$$

In such a definition $q$ does not make any contribution to the total energy of the system. Thus, effectively, in four dimensions, the DM solution describes a Reissner-Nordstrom-like black hole, but, unlike an ordinary Reissner-Nordstrom metric, is asymptotically AdS. As the $f(r)$ metric function in Reissner-Nordstrom-AdS solution in GR has the following form:

$$
f(r)=1-\frac{2 G M}{c^{2} r}+\frac{G Q^{2}}{4 \pi \epsilon_{0} c^{4} r^{2}}+\frac{r^{2}}{l^{2}},
$$

where $G$ is the gravitational constant, $M$ is the mass of the black hole, $Q$ is the electric charge of the black hole, $l$ is the AdS radius, and the total energy of a Reissner-Nordstrom black hole does not obtain any contribution from $Q^{2}$. Thus, it is possible to treat the $M$ as an effective $4 \mathrm{D}$ "mass" and $q$ as an effective "electric charge" in the DM solution. Unlike $Q^{2}$ in the Reissner-Nordstrom solution, $q$ in the DM one can take both positive and negative values. In addition, it was also shown that the DM solution is stable both in an axially-symmetric case and without symmetries [38]. Therefore, it becomes possible to give the non-contradictory definition of a total mass in the DM solution. As this solution is stable, there is a preliminary possibility that such solution could describe a real astrophysical object.

Different combinations of $M$ and $q$ are shown in Figure 5 where negative and positive branches of (22) are denoted as $f_{-}$and $f_{+}$, respectively. Since the total energy of the system must be positive or equal to zero, the $M$ parameter should be non-negative in $f_{-}$and non-positive in $f_{+}$. The horizon radius can be defined as a function upon $r$ when (22) vanishes, thus only the negative branch allows the existence of horizon(s), whereas the positive one can describe only naked singularities. The curve in the 
$M>0$ area is the "one-horizon curve", where $f_{-}$vanishes at one point. The parametric representation of this curve is:

$$
\left\{\begin{array}{l}
M=\frac{s^{3}}{12}+s, \\
q=\frac{s^{4}}{16}+\frac{s^{2}}{2}-1,
\end{array}\right.
$$

where $s \geq 0$. The positive $q$ area under the curve corresponds to the case when the metric is well-defined only outside the horizon(s). The restriction for $M<0$ in the positive branch appears to be as [38]:

$$
q \leq-\frac{1}{8}(6|M|)^{\frac{4}{3}}
$$

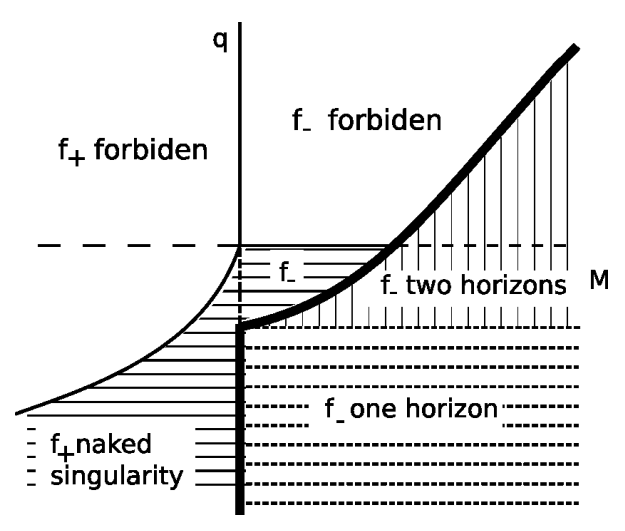

Figure 5. Dadhich-Molina solution phase diagram. Reproduced from [38].

The $M=0$ case represents a naked singularity (if $-1<q<0$ ) or a black hole with one horizon (if $q \leq-1$ ) with a gravitational potential fall-off $\propto r^{-2}$ like an electric potential in (25). Since all observable macroscopic objects in the Universe are electrically neutral, the observation of $\propto r^{-2}$ potential would imply a discovery of a DM-object with $M=0$. This fact makes such an object, at least theoretically, observable. Furthermore, a combination of a $\propto r^{-2}$ potential with an ordinary $\propto r^{-1}$ could help to explain an irregular dynamics in galaxies, being an alternative to the theory of dark matter.

Test particle orbits were studied taking advantage of the analogy between the DM solution in Gauss-Bonnet gravity and the Dadhich-Rezania solution in the Randall-Sundrum II model obtained in [39] and developed in [40]. In order to make the orbital picture consistent with observations [41], one has to require that

$$
|q| \leq \frac{9}{8 \sqrt{6}} M^{2}
$$

Thus, large negative values of $q$ in Figure 5 should also be excluded, as they change the orbital picture significantly from the observed one [38].

Furthermore, the temperature of a DM black hole was calculated with the help of both Hawking [42] and Shankaranarayanan-Padmanabhan-Srinivasan [43] methods. Both lead to similar results. The decreasing of $q$ corresponds to the growth of temperature, analogously to the charged black hole in GR with the decreasing of the electric charge. The temperature-mass dependence for the $q=0$ case is illustrated in Figure 6. Unlike an ordinary Schwarzschild-AdS black hole in GR, the temperature of a DM black hole only increases with growing mass, taking arbitrary positive values. 


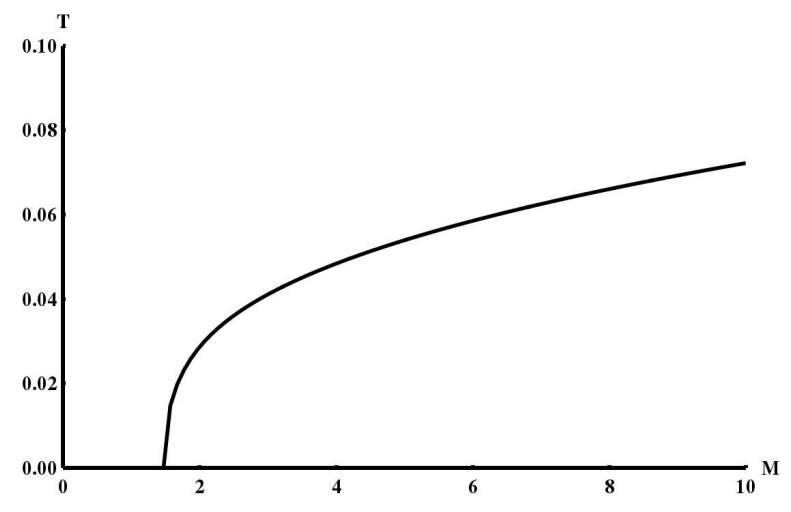

Figure 6. Temperature-mass dependence for Dadhich-Molina black holes in the $q=0$ case. Reproduced from [38].

The decrease of the mass lost rate with mass decreasing implies that the evaporation speed becomes less with time. This is opposite to the usual Hawking evaporation picture in GR. Hence, the lifespan of a DM black hole is estimated to be infinite. Furthermore, black holes that lie on the "one-horizon curve" in the phase diagram in Figure 5 do not evaporate at all. The final stage of the evaporation process depends upon the initial value of the $q$ since it represents the influence of additional dimensions. For $q>-1$, the final stage always lies on the "one-horizon curve" and has a positive "mass". In the $q<-1$ case, the final stage has a zero "mass". The endpoint masses appear to be too small for observations.

\section{Black Hole with the Tidal Charge}

Another model that has the origin in the low-energy effective limit of string theory is a brane world model, where all matter fields are localized on a $(3+1)$-dimensional brane embedded in a higher-dimensional space-time and only gravity can propagate into extra dimensions. In the Randall-Sundrum model [44], an additional non-compact extra dimension exists.

A black hole solution in an RS model was presented by Dadhich, Maartens, Papadopoulos, and Rezania in 2000 [39]. They use the following 5D field equations:

$$
\stackrel{(5)}{G}_{A B}=\stackrel{(5)}{\kappa}^{2}\left[-\stackrel{(5)}{\Lambda}_{\stackrel{(5)}{g}}^{A B}+\delta(\chi)\left(-\lambda \delta_{A}^{\mu} \delta_{B}^{v} g_{\mu v}+\delta_{A}^{\mu} \delta_{B}^{v} T_{\mu v}\right)\right]
$$

where five-dimensional quantities are denoted with "(5)", five-dimensional indexes are Latin capital letters, four-dimensional indexes are Greek letters, $\kappa^{2}=8 \pi / \stackrel{(5)}{M_{p l}^{3}}, \stackrel{(5)}{M_{p l}}$ is a five-dimensional Planck (5)

mass, $\Lambda$ is a five-dimensional cosmological constant, $\lambda$ is the brane tension, $g_{\mu \nu}$ is the induced metric on the brane, and $\chi$ is the fifth dimension coordinate (the brane is located at $\chi=0$ ). As is shown in [45], the induced field equations on the brane have the following form:

$$
G_{\mu v}=-\Lambda g_{\mu v}+\kappa^{2} T_{\mu v}+\stackrel{(5)}{\kappa^{4}} S_{\mu \nu}-\mathcal{E}_{\mu v}
$$

where $\kappa^{2}=8 \pi / M_{p l}{ }^{2}, S_{\mu v}$ is "squared energy-momentum tensor":

$$
S_{\mu v}=-\frac{1}{4} T_{\mu \alpha} T_{\nu}^{\alpha}+\frac{1}{12} T T_{\mu v}+\frac{1}{8} g_{\mu \nu} T_{\alpha \beta} T^{\alpha \beta}-\frac{1}{24} g_{\mu \nu} T^{2} .
$$


$\mathcal{E}_{\mu \nu}$ is the limit on the brane of the projected bulk Weyl tensor:

$$
\mathcal{E}_{\mu \nu}=\delta_{\mu}^{A} \delta_{v}^{B} C_{A C B D}^{(5)} n^{C} n^{D},
$$

(5)

where $C_{A C B D}$ is the 5D Weyl tensor, $n^{C}$ is a vector unit normal to the brane.

As the four-dimensional quantities can be written as functions of five-dimensional ones as:

$$
\begin{aligned}
& M_{p l}=\sqrt{\frac{3}{4 \pi \lambda}} M_{p l}^{3}, \\
& \Lambda=\frac{\frac{4 \pi}{(5)}}{M_{p l}^{3}}\left(\stackrel{(5)}{\Lambda}+\frac{4 \pi \lambda^{2}}{(5)}\right),
\end{aligned}
$$

a spherically-symmetric stationary vacuum solution, obtained in [39], is given by:

$$
d s^{2}=\Delta(r) d t^{2}-\frac{1}{\Delta(r)} d r^{2}-r^{2} d \Omega^{2},
$$

where

$$
\Delta(r)=1-\frac{2 M}{M_{p l}^{2} r}+\frac{q}{M_{p l}^{2} r^{2}} .
$$

$M$ is the mass of the black hole and $q$ is the "tidal charge", coming from the Weyl tensor projection.

The metric function (36) is the same as a Reissner-Nordstrom solution in GR. If $q$ is greater than zero, the solution [39] coincides with the Reissner-Nordstrom one and has two horizons. If $q$ is negative, the solution has a unique horizon with the radius:

$$
r_{h}=\frac{M}{M_{p l}^{2}}\left(1+\sqrt{1-q \frac{M_{p l}^{4}}{M^{2} M_{p l}^{2}}}\right) .
$$

Since in the $q<0$ case the effective energy density on the brane is negative, this case looks more physically sensible [39]. In the solar system, the following upper limit upon $q$ is constrained:

$$
|q|<<2\left(\frac{M_{p l}^{(5)}}{M_{p l}}\right)^{2} M_{s o l} R_{s o l}
$$

where $M_{s o l}$ is Solar mass and $R_{\text {sol }}$ is its radius. The geodesics in the Dadhich-Maartens-Papadopoulos-Rezania solution were studied in [46]. The geodesic equations are:

$$
\left\{\begin{array}{l}
\frac{d}{d \tau} t(\tau)-\frac{r^{2}(\tau) E}{r^{2}(\tau)+\alpha r(\tau)+\beta}=0, \\
\left(\frac{d u}{d \phi}\right)^{2}=\frac{E^{2}-1}{L^{2}}-\frac{\alpha u+\beta u^{2}}{L^{2}}-u^{2}-\alpha u^{3}+\beta u^{4}, \\
\frac{d \tau}{d \phi}=\frac{1}{u^{2} l^{2}}, \\
\frac{d t}{d \phi}=\frac{E}{L^{2}\left(u^{2}+\alpha u^{3}+\beta u^{4}\right)}
\end{array}\right.
$$

where $\tau$ is the proper time, $t$ is the coordinate time, $u=\frac{1}{r}, \alpha=-\frac{2 M}{M_{p l}^{2}}$, and $\beta=\frac{q}{M_{p l}^{2}}$. When $r$ has an upper limit, $E^{2}<1$. The right-hand side of the second equation of the system (39) (which we denote as $f(u)$ ) is a fourth-order polynomial with respect to $u$. It is important to emphasise that, in the Schwarzschild solution, it has the third order. Thus, an additional root of $f(u)$ appears. Regardless of $E$ and $L$ values 
for arbitrary orbits, $f(u)$ cannot have four positive roots, since $\alpha / \beta>0$, so there are no new types of finite orbits that do not exist in a Schwarzschild solution.

Radial and circular geodesics are studied separately. In the case of zero angular momentum, the geodesic equation can be written as follows:

$$
\left(\frac{d r}{d \tau}\right)^{2}=E^{2}-\Delta
$$

With the initial conditions $r=r_{i}, \dot{r}=0$, one obtains

$$
r_{i}=\frac{\sqrt{\alpha^{2}-4 \beta\left(1-E^{2}\right)}-\alpha}{2\left(1-E^{2}\right)}
$$

which differs from the corresponding value in Schwarzschild geometry. The proper time necessary to reach the central singularity can be calculated by introducing a new variable $\eta$ :

$$
r=r_{i} \cos ^{2}\left(\frac{\eta}{2}\right)
$$

After all the calculations, one obtains:

$$
\tau=\frac{\sqrt{-\left(1-E^{2}\right) r_{i}^{2} x^{2}-\alpha r_{i} x-2 \beta}}{1-E^{2}}-\frac{\alpha}{2\left(1-E^{2}\right)^{\frac{3}{2}}} \arcsin \left[-\frac{2\left(1-E^{2}\right) r_{i} x+\alpha}{\sqrt{\alpha^{2}-8 \beta\left(1-E^{2}\right)}}\right]-\tau_{1},
$$

where the integration constant $\tau_{1}$ can be determined by requiring $\tau(x=1)=0$. The consideration of the $\beta \rightarrow 0$ limit results in the proper time necessary to reach the central singularity:

$$
\tau^{s c h}=\sqrt{\frac{r_{i}^{3}}{4 \alpha}} \pi
$$

This means that radial geodesics in the Dadhich-Maartens-Papadopoulos-Rezania solution have a Schwarzschild limit.

In order to calculate the time of reaching the horizon in the reference frame of a remote observer, one can use the first equation of system (39), so:

$$
\frac{d t}{d r}=\frac{-E r^{3}}{\left(2 r_{h}+\alpha\right) \sqrt{-\left(1-E^{2}\right) r^{2}-\alpha r-2 \beta}}\left(\frac{1}{r-r_{h}}-\frac{1}{r+r_{h}+\alpha}\right) .
$$

The integral diverges at the horizon, analogously to the one in Schwarzschild solution. Thus, the presence of the "tidal charge" only quantitatively alter the solution, but the behaviour of a particle moving on a radial geodesic remains similar to GR.

For a circular orbit [46],

$$
\begin{gathered}
E^{2}=\frac{2\left(1+\alpha u_{c}+\beta u_{c}^{2}\right)^{2}}{2+3 \alpha u_{c}+\beta u_{c}^{2}}, \\
L^{2}=\frac{-\alpha-2 \beta u_{c}}{u_{c}\left(2+3 \alpha u_{c}+4 \beta u_{c}^{2}\right)},
\end{gathered}
$$

where $u_{c}$ is the inverse radius of the orbit.

For a nonzero $\beta, L^{2}$ is bigger than in the Schwarzschild case. Since $E^{2}, L^{2}$ are positive, the denominator must also be positive:

$$
2+3 \alpha u_{c}+4 \beta u_{c}^{2}>0
$$


For a negative $\beta$, one obtains:

$$
\begin{aligned}
& 0<u_{c}<\frac{-3 \alpha+\sqrt{9 \alpha^{2}-32 \beta}}{8 \beta}, \\
& \frac{-3 \alpha+\sqrt{9 \alpha^{2}-32 \beta}}{4}<r_{c}<\infty .
\end{aligned}
$$

The last stable orbit inverse radius is the inflection point of the right-hand side of this equation and can be determined from the following equation:

$$
8 \beta^{2} u_{c}^{3}+2 \alpha u_{c}^{2}+3 \alpha^{2} u_{c}+\alpha=0
$$

where $u_{c}$ is the inverse radius of the last stable orbit. In the $\beta \rightarrow 0$ limit, one obtains the Schwarzschild case. The terms containing $\beta$ have to be negligible at the scale of $M_{\text {sol }}$ order and larger in order to be consistent with black hole observations, which agree with Schwarzschild [47]. Additional restrictions upon $\beta$ can arise from this requirement [46]. It is convenient to represent $\alpha$ and $\beta$ in the following form:

$$
\alpha=-a \frac{M_{s o l}}{M_{p l}^{2}}, \quad \beta=b \frac{M_{s o l}^{2}}{M_{p l}^{4}},
$$

where $a \sim 1$. Then, a new variable can be introduced

$$
\tilde{u}_{c}=u_{c} \frac{M_{s o l}}{M_{p l}^{2}},
$$

and (51) is rewritten as follows:

$$
8 b^{2} \tilde{u}_{c}^{3}+9 a b \tilde{u}_{c}^{2}+3 a^{2} \tilde{u}_{c}+a=0
$$

Requiring that the terms with "tidal charge" should be negligibly small in comparison with the Schwarzschild ones results in the following restriction:

$$
|b| \ll 1
$$

In this parameter range $(a \sim 1,|b| \ll 1)$, the presence of the "tidal charge" does not alter the types and structure of geodesics. Since for solar and bigger than solar masses of the black hole the solution does not differ from Schwarzschild, no observable effects can make the solution distinguishable, although in the microphysics the difference could be detectable [46]. Additional constraints on the "tidal charge" could be placed by considering circular orbits and the black hole shadows $[48,49]$.

\section{Brans-Dicke Theory}

Brans-Dicke theory appeared in 1961 [50], initially as a relativistic theory of gravitation, compatible with Mach's principle, generalising GR. The core idea was to consider the gravitational constant $G$ as a function upon a certain scalar field rather than a constant. Thus, a new scalar field $\phi$ was included into the gravitational action:

$$
S=\frac{1}{2 \kappa} \int d^{4} x \sqrt{-g}\left(\phi R-\omega \frac{\partial_{\mu} \phi \partial^{\mu} \phi}{\phi}\right),
$$

where $\omega$ is the dimensionless Brans-Dicke parameter. The limit $\omega \rightarrow \infty$ gives GR. The observational bound on $\omega$ is [51]:

$$
|\omega|>50,000 .
$$


The introduction of a massive scalar field makes it possible to describe the dark energy, as the evolution of the Universe in Brans-Dicke theory could mimic the $\Lambda$ CDM model [52]. Various interesting spherically-symmetric solutions were obtained in the massless version $(56)[50,53-55]$ and in the extended ones [56,57].

\subsection{Brans-Dicke Spherically-Symmetric Wormhole}

The first static spherically-symmetric solution in the framework of Brans-Dicke theory was obtained by Brans and Dicke themselves in their original paper [50]. The field equations, deduced from the gravitational action of the form (56), are

$$
G_{\mu v}=\frac{8 \pi}{\phi c^{4}} T_{\mu v}+\frac{\omega}{\phi^{2}}\left(\partial_{\mu} \phi \partial_{\nu} \phi-\frac{1}{2} g_{\mu \nu} \partial_{\rho} \phi \partial^{\rho} \phi\right)+\frac{1}{\phi}\left(\nabla_{\mu} \nabla_{\nu} \phi-g_{\mu v} \square \phi\right) .
$$

The Klein-Gordon equation for $\phi$ has the form:

$$
\square \phi=\frac{8 \pi}{(3+2 \omega) c^{4}} T .
$$

The Brans-Dicke (BD) solution has the following form [58]:

$$
d s^{2}=-\left(\frac{1-1 / x}{1+1 / x}\right)^{2 l} c^{2} d t^{2}+\left(1+\frac{1}{x}\right)^{4}\left(\frac{1+1 / x}{1-1 / x}\right)^{n}\left(d r^{2}+r^{2} d \Omega^{2}\right),
$$

where

$$
\phi=A\left(\frac{1-1 / x}{1+1 / x}\right)^{p}
$$

$x=\frac{r}{B}, l=\frac{1}{\lambda}, n=\frac{\lambda-C-1}{\lambda}, p=\frac{C}{\lambda}$,

$$
\lambda=\sqrt{\frac{2 \omega+3}{2 \omega+4}}, \quad B=\frac{M}{2 A c^{2}} \sqrt{\frac{2 \omega+4}{2 \omega+3}}, \quad C=-\frac{1}{\omega+2},
$$

where $M$ is the asymptotic mass of the solution.

This solution describes a possibly traversal wormhole with the scalar field $\phi$ playing the role of exotic matter. The radius of the throat can be calculated using the following formula [58]:

$$
\begin{aligned}
r_{0}= & \frac{\sqrt{2} B}{2} \frac{2|\omega+1| \pm(\omega+2) \sqrt{\frac{-8-6 \omega}{(\omega+2)^{2}}}}{\sqrt{(2 \omega+3)(\omega+2)}}\left(1+\frac{\sqrt{2}(\omega+2) \sqrt{\frac{2 \omega+3}{\omega+2}}}{2(\omega+1) \pm(\omega+2) \sqrt{-\frac{8+6 \omega}{(\omega+2)^{2}}}}\right)^{2} \\
& \times\left(\frac{1-\frac{\sqrt{2}(\omega+2) \sqrt{\frac{2 \omega+3}{\omega+2}}}{2(\omega+1) \pm(\omega+2) \sqrt{-\frac{8+6 \omega}{(\omega+2)^{2}}}}}{1+\frac{\sqrt{2}(\omega+2) \sqrt{\frac{2 \omega+3}{\omega+2}}}{2(\omega+1) \pm(\omega+2) \sqrt{-\frac{8+6 \omega}{(\omega+2)^{2}}}}}\right)^{1+\frac{1}{\omega+2} \sqrt{\frac{2(\omega+2)}{2 \omega+3}}-\sqrt{\frac{2(\omega+2)}{2 \omega+3}}} .
\end{aligned}
$$

Within the possible parameter range (57), the radius appears to be almost exactly equal to the horizon radius of a Schwarzschild black hole:

$$
r_{0} \approx \frac{2 G M}{c^{2}} .
$$


Most of the astrophysical objects have accretion disks. In order to calculate the flux of energy from the accretion disk around the wormhole, the movement of particles near the throat must be studied. The energy of the particle is given by

$$
\tilde{E}=\left(\frac{c-\lambda}{x+\lambda}\right)^{l} \sqrt{\frac{x^{2}+\lambda^{2}-2 x(C+1)}{x^{2}-\lambda^{2}-2 x(C+2)}}
$$

the angular momentum is

$$
\tilde{L}=\sqrt{\frac{2}{x}} B \frac{x^{2}-\lambda^{2}}{\sqrt{x^{2}+\lambda^{2}-2 x(C+2)}}\left(\frac{x+\lambda}{x-\lambda}\right)^{l+p}
$$

and the angular velocity is

$$
\Omega=\frac{x}{B} \frac{1}{x^{2}-\lambda^{2}} \sqrt{\frac{2 x}{x^{2}+\lambda^{2}-2 x(C+1)}}\left(\frac{x-\lambda}{x+\lambda}\right)^{p+2 l} .
$$

Therefore, the radius of the last stable orbit is [58]:

$$
r_{m s} \approx \frac{5 G M}{c^{2}} .
$$

The flux of energy from a flat accretion disk can be determined as follows:

$$
F(r)=-\frac{G \dot{M}_{0}}{4 \pi c^{2} \sqrt{-g}} \frac{\partial_{r} \Omega}{(\tilde{E}-\Omega \tilde{L})^{2}} \int_{r_{m s}}^{r}(\tilde{E}-\Omega \tilde{L}) \partial_{r} \tilde{L} d r .
$$

By substituting (65)-(68) into (69), it is possible to calculate the flux. The result is presented in Figure 7 as a function of $r / M$. The flux from the accretion disk around a BD-wormhole turns out to be considerably smaller than from a GR wormhole (exceeding $4 \times 10^{-22} \mathrm{erg} \mathrm{cm}^{-2} \mathrm{~s}^{-1}$ ), but almost indistinguishable from the one around an ordinary GR Schwarzschild black hole.

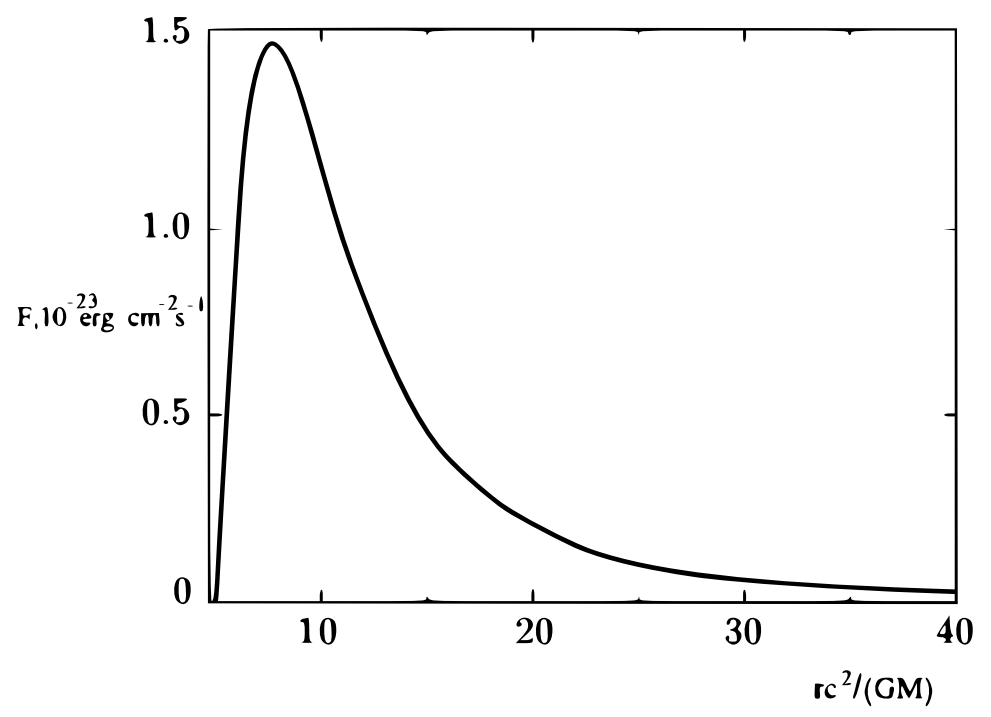

Figure 7. The energy flux from an accretion disk around a BD-wormhole. Reproduced from [58].

The maximal impact parameter, from which the observer could see the light coming through the wormhole is

$$
h_{\max } \approx 5.18 \frac{G M}{c^{2}},
$$


which makes it possible to distinguish a BD-wormhole from a GR wormhole,for which $h_{\max } \approx 4 \frac{G M}{c^{2}}$ [58].

\subsection{Wormhole Embedded in a de Sitter Universe Solution}

Furthermore, a static spherically-symmetric solution in the framework of Brans-Dicke theory with an exponential potential [56] was presented [59]. The corresponding vacuum field equations are:

$$
G_{\mu \nu}=\omega\left(\partial_{\mu} \phi \partial_{\nu} \phi-\frac{1}{2} g_{\mu \nu} \partial_{\rho} \phi \partial^{\rho} \phi\right)+e^{-\alpha \phi}\left(\nabla_{\mu} \nabla v e^{\alpha \phi}-g_{\mu \nu} \square e^{\alpha \phi}\right)-\frac{1}{2} g_{\mu \nu} V_{0} e^{\frac{\phi}{\phi_{0}}},
$$

and Klein-Gordon equations [53]:

$$
\alpha R+\omega \alpha \partial_{\mu} \phi \partial^{\mu} \phi-V_{0} e^{\frac{\phi}{\phi_{0}}}\left(\alpha+\frac{1}{\phi_{0}}\right)+2 \omega \square \phi=0 .
$$

After substitutions, the field equations take the form:

$$
R_{\mu \nu}=\omega \partial_{\mu} \phi \partial_{\nu} \phi+e^{-\alpha \phi} \nabla_{\mu} \nabla v e^{\alpha \phi}+\frac{1}{2} g_{\mu \nu} D e^{\frac{\phi}{\phi_{0}}}
$$

where $R_{\mu v}$ is the Ricci tensor,

$$
D=V_{0}\left[1-\left(\alpha-\frac{1}{\phi_{0}}\right) \frac{\alpha}{2 \omega+3 \alpha^{2}}\right] .
$$

A static spherically-symmetric metric can be written in GM-GHS gauge:

$$
d s^{2}=\Delta d t^{2}-\frac{1}{\Delta} d r^{2}-R(r)^{2} d \Omega^{2},
$$

where $\Delta$ and $R$ are functions of $r$. After substituting this metric into Equation (73), it decomposes into the following system:

$$
\left\{\begin{array}{l}
\Delta^{\prime \prime}=\frac{D R e^{\frac{\phi}{\phi_{0}}}-2 \Delta^{\prime} R^{\prime}}{R}, \\
R^{\prime \prime}=\frac{-2 R \Delta^{\prime} R^{\prime}-2 \Delta\left(R^{\prime}\right)^{2}+D R^{2} e^{\frac{\phi}{\phi_{0}}}+2}{2 \Delta R}, \\
\phi^{\prime \prime}=\frac{\left(\alpha^{2}+\omega\right)\left(\phi^{\prime}\right)^{2}}{\alpha}+\frac{2\left[\Delta\left(R^{\prime}\right)^{2}-1\right]}{\alpha \Delta R^{2}}-\frac{D R e^{\frac{\phi}{\phi_{0}}}-2 \Delta^{\prime} R^{\prime}}{\alpha \Delta R} .
\end{array}\right.
$$
leads to:

The requirement that, in the limit $r \rightarrow \infty$, the potential term tends to the cosmological constant

$$
\begin{aligned}
V_{0} & =2 \Lambda e^{\frac{-\phi_{\infty}}{\phi_{0}}} \\
\phi_{\infty} & =\frac{1}{\alpha} \ln \left[\frac{1}{G_{0}} \frac{2 \omega+4 \alpha^{2}}{2 \omega+3 \alpha^{2}}\right]
\end{aligned}
$$

where $\Lambda$ is the cosmological constant.

The numerical solution of (76) describes a wormhole embedded in a de Sitter universe if $22.7 \leq \phi_{0} \leq 25$, a naked singularity in a de Sitter universe if $\phi_{0}<22.7$, and tends to Schwarzschild black hole with $\phi_{0}$ increasing [59].

Figure 8 illustrates the behaviour of the metric function $\Delta$. Near the throat, at astrophysical distances, the metric is wormhole-like, whereas, at cosmological distances, the geometry tends to dS. Thus, the solution is capable of describing both astrophysical objects and cosmological ones. 


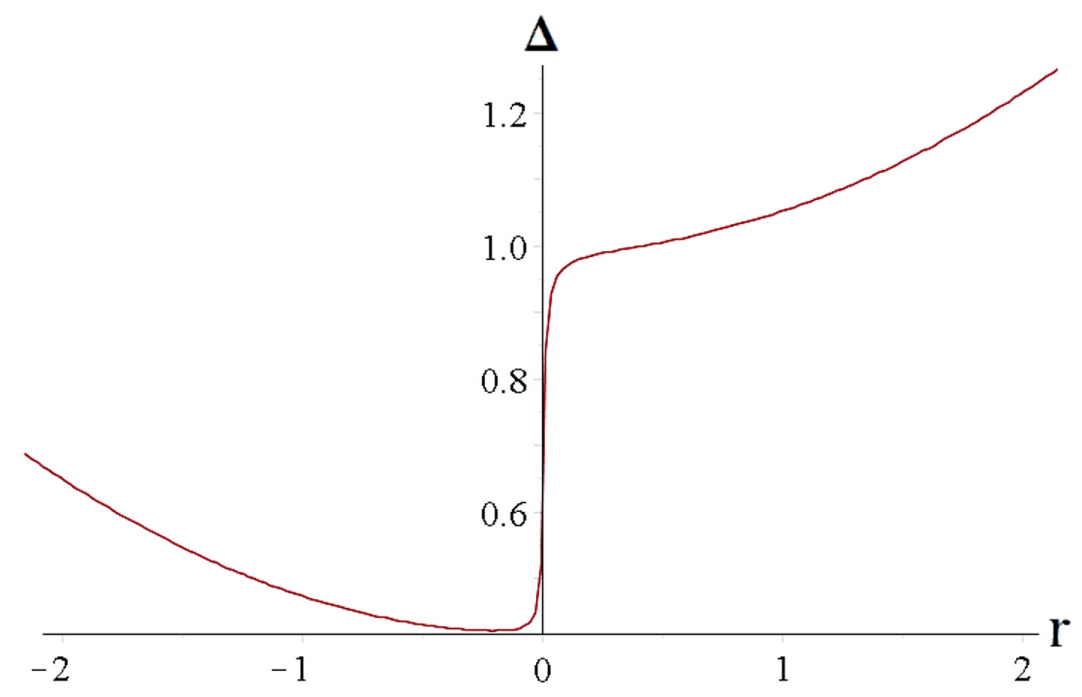

Figure 8. The metric function $\Delta$ of the wormhole solution in a de Sitter universe, $\phi_{0}=24.5$. Reproduced from [59].

As the behaviour of the metric function $\Delta$ and the scalar field $\phi$ is obtained, it becomes possible to calculate the throat radius and, for $\phi_{0}=24.5$, it is [59]

$$
r_{0} \approx 5927 \frac{G M}{c^{2}}
$$

which is considerably bigger than the radius of the throat without the potential $\left(r_{0} \approx 3014 \frac{G M}{c^{2}}\right)$. Depending on the value of $\phi_{0}$, the difference between the metric function $\Delta$ in Schwarzschild solution and the obtained one can be rather large. For instance, the value of $\Delta$ calculated at the point of the last stable orbit:

$$
\frac{\Delta}{\Delta_{\text {schw }}} \approx 1.031
$$

for $\phi_{0}=24.5$, and

$$
\frac{\Delta}{\Delta_{\text {schw }}} \approx 1.471
$$

for $\phi_{0}=23.5$, where $\Delta_{\text {schw }}$ is the Schwarzschild metric function. Hence, future black hole observations can place additional constraints on the $\phi_{0}$ parameter.

\section{Discussion}

In this paper, we discussed a set of black hole and wormhole solutions appearing in Brans-Dicke and Gauss-Bonnet models. It is important to note that models with higher order curvature corrections firstly appeared in the attempts to construct the theory of quantum gravity. Furthermore, such expansions became a part of a string gravity effective action. The solutions obtained at this stage could expand the known GR ones but only in the high energy region where the curvature began to diverge. As these solutions can not help in solving dark energy and dark matter problems, the development of extended models went on. When the ideas of non-compact extra dimensions were taken into account, the possibility to describe the astrophysical objects and processes became more realistic. This approach appeared to be very fruitful and remains actual now because of the hope to describe black hole shadows that were discovered not so long ago [60]. The community is waiting for the accuracy to increase [61].

The Brans-Dicke model now serves as a first step at each attempt of GR extension [1]. Its scalar field often presents a possibility to describe particularities from more complicated models [62]. This is why the solutions in the Brans-Dicke model represent interest by themselves. Wormhole solutions 
are less studied than black hole ones so, even at the level of the Brans-Dicke model, one can hope to extract something new for these objects as we tried to demonstrate based on a few papers.

Finally, we hope that the methods used while studying these models would also be useful in further investigations of extended gravity models.

Author Contributions: The text is based mostly on S.A. and his co-authors old results, so, the role of S.A. was conceptualization, data curation, supervision and final editing. The role of M.S. is validation, visualisation and writing of the original draft. All authors have read and agree to the published version of the manuscript.

Funding: The authors acknowledge the support from the program of development of M.V. Lomonosov Moscow State University (Leading Scientific School 'Physics of stars, relativistic objects and galaxies').

Acknowledgments: S.A. would like to kindly thank his co-authors of the papers used for this presentation, especially Michael Pomazanov, Michael Sazhin, Aurelien Barrau, Kristina Rannu, Darya Tretyakova, and Boris Latosh.

Conflicts of Interest: The authors declare no conflict of interest.

\section{References}

1. Tamaki, T.; Maeda, K.; Torii, T. Non-Abelian black holes in Brans-Dicke theory. Phys. Rev. D 1998, 57, 4870-4884. [CrossRef]

2. Novikov, I.D.; Frolov, V.P. Physics of Black Holes; Springer: Dordrecht, The Netherlands, 1989; Volume 27.

3. Lovelock, D. The Einstein tensor and its generalizations. J. Math. Phys. 1971, 12, 498-501. [CrossRef]

4. Lanczos, C. A Remarkable property of the Riemann-Christoffel tensor in four dimensions. Ann. Math. 1938, 39, 842-850. [CrossRef]

5. Higgs, P.W. Quadratic lagrangians and general relativity. Nuovo Cim. 1959, 11, 816-820. [CrossRef]

6. Zwiebach, B. Curvature Squared Terms and String Theories. Phys. Lett. B 1985, 156, 315-317. [CrossRef]

7. Alexeev, S.O.; Pomazanov, M.V. Black hole solutions with dilatonic hair in higher curvature gravity. Phys. Rev. D 1997, 55, 2110-2118. [CrossRef]

8. Kanti, P.; Mavromatos, N.E.; Rizos, J.; Tamvakis, K.; Winstanley, E. Dilatonic black holes in higher curvature string gravity. Phys. Rev. D 1996, 54, 5049-5058. [CrossRef]

9. Wiltshire, D.L. Spherically Symmetric Solutions of Einstein-maxwell Theory With a Gauss-Bonnet Term. Phys. Lett. B 1986, 169, 36-40. [CrossRef]

10. Mignemi, S.; Wiltshire, D.L. Black holes in higher derivative gravity theories. Phys. Rev. D 1992, 46, 1475-1506. [CrossRef] [PubMed]

11. Mignemi, S.; Stewart, N.R. Charged black holes in effective string theory. Phys. Rev. D 1993, 47, 5259-5269. [CrossRef]

12. Alexeyev, S.O.; Rannu, K.A. Gauss-bonnet black holes and possibilities for their experimental search. J. Exp. Theor. Phys. 2012, 114, 406-427. [CrossRef]

13. Alekseev, S.O.; Sazhin, M.V. Four-dimensional dilatonic black holes in Gauss-Bonnet extended string gravity. Gen. Rel. Grav. 1998, 30, 1187-1201.

14. Bakopoulos, A.; Antoniou, G.; Kanti, P. Novel Black-Hole Solutions in Einstein-Scalar-Gauss-Bonnet Theories with a Cosmological Constant. Phys. Rev. D 2019, 99, 064003. [CrossRef]

15. Alexeyev, S.; Mignemi, S. Black holes and naked singularities in low-energy limit of string gravity with modulus field. Class. Quant. Grav. 2001, 18, 4165-4178. [CrossRef]

16. Alexeyev, S.; Monong, Y. Additional Constraints on Coupling Functions for String Gravity with Second Order Curvature Corrections; Nova Science: Hauppauge, NY, USA, 2017; pp. 219-226.

17. Alexeyev, S.; Barrau, A.; Rannu, K.A. Internal structure of a Maxwell-Gauss-Bonnet black hole. Phys. Rev. D 2009, 79, 067503. [CrossRef]

18. Garfinkle, D.; Horowitz, G.T.; Strominger, A. Charged black holes in string theory. Phys. Rev. D 1991, 43, 3140. [CrossRef] [PubMed]

19. Alexeyev, S.; Barrau, A.; Boudoul, G.; Khovanskaya, O.; Sazhin, M. Black hole relics in string gravity: Last stages of Hawking evaporation. Class. Quant. Grav. 2002, 19, 4431-4444. [CrossRef]

20. Minamitsuji, M.; Ikeda, T. Scalarized black holes in the presence of the coupling to Gauss-Bonnet gravity. Phys. Rev. D 2019, 99, 044017. [CrossRef] 
21. Doneva, D.D.; Yazadjiev, S.S. New Gauss-Bonnet Black Holes with Curvature-Induced Scalarization in Extended Scalar-Tensor Theories. Phys. Rev. Lett. 2018, 120, 131103. [CrossRef]

22. Doneva, D.D.; Staykov, K.V.; Yazadjiev, S.S. Gauss-Bonnet black holes with a massive scalar field. Phys. Rev. D 2019, 99, 104045. [CrossRef]

23. Antoniou, G.; Bakopoulos, A.; Kanti, P. Black-Hole Solutions with Scalar Hair in Einstein-Scalar-Gauss-Bonnet Theories. Phys. Rev. D 2018, 97, 084037. [CrossRef]

24. Antoniou, G.; Bakopoulos, A.; Kanti, P. Evasion of No-Hair Theorems and Novel Black-Hole Solutions in Gauss-Bonnet Theories. Phys. Rev. Lett. 2018, 120, 131102. [CrossRef] [PubMed]

25. Bakopoulos, A.; Kanti, P.; Pappas, N. On the Existence of Solutions with a Horizon in Pure Scalar-Gauss-Bonnet Theories arXiv 2019, arXiv:1910.14637.

26. Alekseev, S.O.; Khovanskaya, O.S. Additional study of a restriction on the minimum black hole mass in string gravity. Grav. Cosmol. 2000, 6, 14-18.

27. Aguilar-Pérez, G.; Cruz, M.; Lepe, S.; Moran-Rivera, I. Hairy black hole stability under odd parity perturbations in the Einstein-Gauss-Bonnet model. arXiv 2019, arXiv:1907.06168.

28. Myung, Y.S.; Zou, D.C. Black holes in Gauss-Bonnet and Chern-Simons-scalar theory. arXiv 2019, arXiv:1903.08312.

29. Blázquez-Salcedo, J.L.; Doneva, D.D.; Kunz, J.; Yazadjiev, S.S. Radial perturbations of the scalarized Einstein-Gauss-Bonnet black holes. Phys. Rev. D 2018, 98, 084011. [CrossRef]

30. Cai, R.G. Gauss-Bonnet black holes in AdS spaces. Phys. Rev. D 2002, 65, 084014. [CrossRef]

31. Barrau, A.; Grain, J.; Alexeyev, S.O. Gauss-Bonnet black holes at the LHC: Beyond the dimensionality of space. Phys. Lett. B 2004, 584, 114. [CrossRef]

32. Alexeyev, S.; Popov, N.; Startseva, M.; Barrau, A.; Grain, J. Kerr-Gauss-Bonnet Black Holes: Exact Analytical Solution. J. Exp. Theor. Phys. 2008, 106, 709-713. [CrossRef]

33. Page, D.N. Particle Emission Rates from a Black Hole. 2. Massless Particles from a Rotating Hole. Phys. Rev. D 1976, 14, 3260-3273. [CrossRef]

34. Maeda, H.; Dadhich, N. Kaluza-Klein black hole with negatively curved extra dimensions in string generated gravity models. Phys. Rev. D 2006, 74, 021501. [CrossRef]

35. Maeda, H.; Dadhich, N. Matter without matter: Novel Kaluza-Klein spacetime in Einstein-Gauss-Bonnet gravity. Phys. Rev. D 2007, 75, 044007. [CrossRef]

36. Dadhich, N.; Maeda, H. Origin of matter out of pure curvature. Int. J. Mod. Phys. D 2008, 17, 513-518. [CrossRef]

37. Molina, A.; Dadhich, N. On Kaluza-Klein spacetime in Einstein-Gauss-Bonnet gravity. Int. J. Mod. Phys. D 2009, 18, 599-611. [CrossRef]

38. Alexeyev, S.O.; Petrov, A.N.; Latosh, B.N. Maeda-Dadhich Solutions as Real Black Holes. Phys. Rev. D 2015, 92, 104046. [CrossRef]

39. Dadhich, N.; Maartens, R.; Papadopoulos, P.; Rezania, V. Black holes on the brane. Phys. Lett B. 2000, 487, 1-6. [CrossRef]

40. Pugliese, D.; Quevedo, H.; Ruffini, R. Circular motion of neutral test particles in Reissner-Nordstrom spacetime. Phys. Rev. D 2011, 83, 024021. [CrossRef]

41. Doeleman, S.S.; Fish, V.L.; Schenck, D.E.; Beaudoin, C.; Blundell, R.; Bower, G.C.; Broderick, A.E.; Chamberlin, R.; Freund, R.; Friberg, P.; et al. Jet Launching Structure Resolved Near the Supermassive Black Hole in M87. Science 2012, 338, 355-358. [CrossRef]

42. Hawking, S.W. Particle Creation by Black Holes. Commun. Math. Phys. 1975, 43, 199-220. [CrossRef]

43. Shankaranarayanan, S.; Padmanabhan, T.; Srinivasan, K. Hawking radiation in different coordinate settings: Complex paths approach. Class. Quant. Grav. 2002, 19, 2671-2688. [CrossRef]

44. Randall, L.; Sundrum, R. An Alternative to compactification. Phys. Rev. Lett. 1999, 83, 4690-4693. [CrossRef]

45. Shiromizu, T.; Maeda, K.i.; Sasaki, M. The Einstein equation on the 3-brane world. Phys. Rev. D 2000, 62, 024012. [CrossRef]

46. Alexeyev, S.O.; Starodubtseva, D.A. Black holes in models with noncompact extra dimensions. J. Exp. Theor. Phys. 2010, 111, 576-581. [CrossRef]

47. Cherepashchuk, A.M. Black holes in binary stellar systems and galactic nuclei. Phys. Usp. 2014, 57, 359-376. [CrossRef] 
48. Zakharov, A.F. Constraints on a charge in the Reissner-Nordstrom metric for the black hole at the Galactic Center. Phys. Rev. D 2014, 90, 062007. [CrossRef]

49. Alexeyev, S.O.; Latosh, B.N.; Prokopov, V.A.; Emtsova, E.D. Phenomenological Extension for Tidal Charge Black Hole. J. Exp. Theor. Phys. 2019, 128, 720-726. [CrossRef]

50. Brans, C.; Dicke, R.H. Mach's principle and a relativistic theory of gravitation. Phys. Rev. 1961, 124, 925-935. [CrossRef]

51. Bertotti, B.; Iess, L.; Tortora, P. A test of general relativity using radio links with the Cassini spacecraft. Nature 2003, 425, 374-376. [CrossRef]

52. Hrycyna, O.; Szydlowski, M. Brans-Dicke theory and the emergence of $\Lambda$ CDM model. Phys. Rev. D 2013, 88, 064018. [CrossRef]

53. Campanelli, M.; Lousto, C.O. Are black holes in Brans-Dicke theory precisely the same as a general relativity? Int. J. Mod. Phys. D 1993, 2, 451-462. [CrossRef]

54. Agnese, A.G.; La Camera, M. Wormholes in the Brans-Dicke theory of gravitation. Phys. Rev. D 1995, 51, 2011-2013. [CrossRef] [PubMed]

55. Bhadra, A.; Sarkar, K. On static spherically symmetric solutions of the vacuum Brans-Dicke theory. Gen. Relativ. Gravit. 2005, 37, 2189-2199. [CrossRef]

56. Elizalde, E.; Nojiri, S.; Odintsov, S.D. Late-time cosmology in (phantom) scalar-tensor theory: Dark energy and the cosmic speed-up. Phys. Rev. D 2004, 70, 043539. [CrossRef]

57. Xiao, X.G.; Zhu, J.Y. Wormhole solution in vacuum Brans-Dicke theory with cosmological constant. Chin. Phys. Lett. 1996, 13, 405-408. [CrossRef]

58. Alexeyev, S.O.; Rannu, K.A.; Gareeva, D.V. Possible observation sequences of Brans-Dicke wormholes. J. Exp. Theor. Phys. 2011, 113, 628-636. [CrossRef]

59. Tretyakova, D.A.; Latosh, B.N.; Alexeyev, S.O. Wormholes and naked singularities in Brans-Dicke cosmology. Class. Quant. Grav. 2015, 32, 18500. [CrossRef]

60. Akiyama, K.; Alberdi, A.; Alef, W.; Asada, K.; Azulay, R.; Baczko, A.-K.; Ball, D.; Baloković, M.; Barrett, J.; Bintley, D.; et al. First, M87 Event Horizon Telescope Results. I. The Shadow of the Supermassive Black Hole. Astrophys. J. Lett. 2019, 875, L1.

61. Goddi, C.; Crew, G.; Impellizzeri, V.; Martí-Vidal, I.; Matthews, L.D.; Messias, H.; Rottmann, H.; Alef, W.; Blackburn, L.; Bronzwaer, T.; et al. First, M87 Event Horizon Telescope Results and the Role of ALMA. Messenger 2019, 177, 25.

62. Capozziello, S.; De Laurentis, M. Extended Theories of Gravity. Phys. Rep. 2011, 509, 167-321. [CrossRef] 